\title{
The Association between Atopic Dermatitis and Depressive Symptoms in Korean Adults: The Fifth Korea National Health and Nutrition Examination Survey, 2007-2012
}

\author{
Sewon Kim, Jee-Yon Lee, Jin-Young Oh, Lan Chekal, Duk Chul Lee* \\ Department of Family Medicine, Yonsei University College of Medicine, Seoul, Korea
}

\begin{abstract}
Background: Atopic dermatitis (AD) is characterized by pruritic and eczematous skin lesions, which often cause depressive symptoms, anxiety, stress, sleep disturbances, social withdrawal, and stigmatization.

Methods: In total, 23,442 subjects ( $434 \mathrm{AD}$ patients and 23,008 control subjects) aged 19 years or older and without a history of major medical illness or depressive disorders were selected from The Fifth Korea National Health and Nutrition Examination Survey 2007-2012. Following the initial selection, 2,170 age- and sex-matched control subjects were selected using 1:5 propensity score matching. Multiple logistic regression analysis was performed to identify the presence of depressive symptoms of at least 2 weeks in duration.

Results: The demographic, socioeconomic, and clinical characteristics of AD patients and control subjects were presented and compared, and some variables differed significantly between groups. Presence of depressive symptoms was set as dependent variable, and multiple logistic regression analysis was performed as follows: (1) unadjusted; (2) with alcohol use, exercise status, smoking status, and body mass index (BMI) adjusted for; and (3) with alcohol use, exercise status, smoking status, marital status, occupation, BMI, total caloric intake, history of hypertension, and history of diabetes mellitus adjusted for. Depressive symptoms were significantly higher (odds ratios, $1.46,1.40$, and 1.36; 95\% confidence intervals, 1.09-1.95, 1.0.4-1.88, and 1.01-1.85, respectively) in AD patients relative to those of matched controls.

Conclusion: $\mathrm{AD}$ and clinical depression interact closely, and causal relationships between the two conditions have frequently been observed. Physicians should consider mental health interventions cautiously. It is particularly important that primary care physicians provide comprehensive, continuous long-term care.
\end{abstract}

Keywords: Atopic Dermatitis; Depression; Korea National Health and Nutrition Examination Survey; Propensity Score; Logistic Regression 


\section{INTRODUCTION}

Atopic dermatitis (AD) is currently one of the most prevalent chronic relapsing skin diseases diagnosed and treated by primary care physicians nationally in South Korea and also globally. It is characterized by pruritic and eczematous skin lesions, ${ }^{1)}$ which often cause depressive symptoms, anxiety, stress, and sleep and psychological disturbances such as social withdrawal and stigmatization. The age of onset is between 1 year in approximately $50 \%$ of patients and 5 years in approximately $90 \%$ of patients, and in most cases, the skin lesions resolve before the patient reaches the age of 12 years. ${ }^{2)}$ The lesions persist throughout adolescence and early adulthood in less than $10 \%$ of patients, who demonstrate lower levels of treatment compliance and adherence, poorer quality of life, and greater psychosocial disturbance, relative to that of younger patients.

Recently, the need to strengthen patient education and adopt a multifaceted approach to the management of chronic relapsing skin diseases has been emphasized in international studies, because the area of science known as psychodermatology is evolving. ${ }^{3)}$ According to the categorization outlined in psychodermatology, $\mathrm{AD}$ is a psychophysiological disorder, and while stress does not cause $\mathrm{AD}$, it could be precipitate or exacerbate the condition, and emotional factors determine the natural course of the disease. ${ }^{3)}$

Few studies have been conducted to examine the association between $\mathrm{AD}$ and clinically significant depressive symptoms in Korean adults in multicenter settings on a national scale. Therefore, we sought to clarify the similarities and differences, with respect to demographic, socioeconomic, and clinical characteristics, between $\mathrm{AD}$ patients and control subjects in the Fifth Korea National Health and Nutrition Examination Survey 20072012 (KNHANES) and conducted multiple logistic regression analysis to determine whether there was a statistically significant difference in clinically significant depressive symptoms of at least 2 weeks in duration between the two groups. In addition, we sought to discuss means via which primary care physicians could improve $\mathrm{AD}$ management. This cross-sectional study was the first to examine the association between $\mathrm{AD}$ and mental health using KNHANES data for sufficient numbers of adult patients and control subjects.

\section{METHODS}

\section{Subjects}

Initially, 23,442 subjects were selected from the KNHANES according to the following selection criteria: age of 19 years or older and no history of cancer, stroke, cardiovascular disease, tuberculosis, chronic obstructive pulmonary disease, bronchiectasis, chronic kidney disease, thyroid disease, liver disease, or depressive disorders. Of the initial 23,442 subjects, 434 indi- viduals with a current or previous diagnosis of $\mathrm{AD}$ and 2,170 age- and sex-matched control subjects were selected to participate in the study.

\section{Study Variables}

Subjects' demographic characteristics included age, sex, alcohol use, exercise status, and smoking status. Alcohol use was classified as follows: none, up to once per week, and at least twice per week. Exercise status was defined as follows: (1) intensive exercise involving unusual exertion, lasting at least 10 minutes per activity and 20 minutes per day, on at least 3 days during the preceding week or (2) moderate exercise, lasting at least 10 minutes per activity and 30 minutes or longer per day, on at least 5 days during the preceding week. Smoking status was classified as follows: none, past, or current (current smoking was defined as currently smoking, with at least five packs of cigarettes [100 units] smoked during one's lifetime).

Socioeconomic characteristics included residential area, educational level, monthly household income, marital status, and occupation. Educational level was classified according to the highest level of education attained, as follows: (1) elementary school or lower, (2) middle school, (3) high school, and (4) college (undergraduate) or higher. Monthly household income was classified according to quartiles: low ( $<733$ US dollar [USD]), low medium (733-1,429 USD), medium high (1,429-2,381 USD), and high ( $>2,381$ USD). Occupation was classified as follows: professional, managerial, semiskilled nonmanual, semiskilled manual, partly skilled, unskilled, and none.

Clinical characteristics included body mass index (BMI, kg/ $\mathrm{m}^{2}$ ), waist circumference $(\mathrm{cm})$, total caloric intake (kcal), history of hypertension diagnosed and managed by a physician, and history of diabetes mellitus (DM) diagnosed and managed by a physician. Depressive symptoms were considered present if they had lasted for at least 2 weeks. Data are presented as means \pm standard errors or number (\%).

\section{Statistical Analysis}

Descriptive analyses were conducted twice. The initial analysis included 23,008 control subjects and $434 \mathrm{AD}$ patients. Thereafter, 2,170 age- and sex-matched control subjects were selected via 1:5 propensity score matching and reanalyzed. Study variables that were statistically significant in the descriptive analyses were included as independent variables, with the presence of depressive symptoms of at least 2 weeks in duration as the dependent variable. Multiple logistic regression analyses were performed as follows: (1) unadjusted; (2) with alcohol use, exercise status, smoking status, and BMI adjusted for; and (3) with alcohol use, exercise status, smoking status, marital status, occupation, BMI, total caloric intake, history of hypertension, and history of DM adjusted for. All analyses were performed using SAS ver. 9.3 (SAS Institute Inc., Cary, NC, USA). Results with a 
P-value of $<0.05$ were considered statistically significant.

\section{RESULTS}

$\mathrm{AD}$ patients' and control subjects' demographic (age, sex, alcohol use, exercise status, and smoking status), socioeconomic (residential area, educational level, monthly household income, marital status, and occupation) and clinical (BMI $\left[\mathrm{kg} / \mathrm{m}^{2}\right]$, waist circumference $[\mathrm{cm}]$, total caloric intake [kcal], history of hypertension, and history of DM) characteristics are presented in Table 1. AD patients' mean age (39.69; SE, 15.56) was significantly lower relative to that of control subjects (mean age, 48.71; SE, 16.54). With respect to sex, $\mathrm{AD}$ patients (180 men [41.5\%] and 254 women [58.5\%]) and control subjects (9,274 men [40.3\%] and 13,734 women [59.7\%]) did not differ significantly. To improve the quality of controls and accuracy of descriptive analy-

Table 1. Characteristics of atopic dermatitis patients and control subjects (unweighted data)

\begin{tabular}{|c|c|c|c|c|c|c|}
\hline \multirow[b]{2}{*}{ Variable } & \multicolumn{3}{|c|}{ Before 1:5 matching } & \multicolumn{3}{|c|}{ After 1:5 matching } \\
\hline & $\begin{array}{c}\text { Control subjects } \\
(n=23,008)\end{array}$ & $\begin{array}{l}\text { Atopic dermatitis } \\
\text { patients }(n=434)\end{array}$ & P-value & $\begin{array}{l}\text { Control subjects } \\
\quad(n=2,170)\end{array}$ & $\begin{array}{l}\text { Atopic dermatitis } \\
\text { patients }(n=434)\end{array}$ & P-value \\
\hline \multicolumn{7}{|l|}{ Demographic characteristics } \\
\hline Age (y) & $48.71 \pm 16.54$ & $39.69 \pm 15.56$ & $<0.01$ & $39.69 \pm 15.54$ & $39.69 \pm 15.56$ & 1.00 \\
\hline Sex & & & 0.62 & & & 1.00 \\
\hline Male & $9,274(40.3)$ & $180(41.5)$ & & $900(41.5)$ & $180(41.5)$ & \\
\hline Female & $13,734(59.7)$ & $254(58.5)$ & & $1,270(58.5)$ & $254(58.5)$ & \\
\hline Alcohol use & & & $<0.01$ & & & 0.54 \\
\hline None & $6,324(27.5)$ & $88(20.2)$ & & $448(20.7)$ & $88(20.2)$ & \\
\hline$\leq 1 /$ wk & $11,871(51.6)$ & $265(61.1)$ & & $1,270(58.5)$ & $265(61.1)$ & \\
\hline$\geq 2 / w k$ & $4,813(20.9)$ & $81(18.7)$ & & $452(20.8)$ & $81(18.7)$ & \\
\hline Exercise status & $1,666(7.2)$ & $44(10.1)$ & 0.02 & $178(8.2)$ & $44(10.1)$ & 0.18 \\
\hline Smoking & & & 0.03 & & & 0.31 \\
\hline None & $14,365(62.5)$ & $225(58.8)$ & & 1,354 (62.4) & $255(58.8)$ & \\
\hline Past & 2,285 (9.9) & $35(8.1)$ & & $175(8.1)$ & $35(8.1)$ & \\
\hline Current & 6,358 (27.6) & $144(33.1)$ & & $641(29.5)$ & $144(33.1)$ & \\
\hline \multicolumn{7}{|l|}{ Socioeconomic characteristics } \\
\hline Residential area & & & 0.04 & & & 0.69 \\
\hline Urban & $17,399(75.6)$ & $347(80.0)$ & & $1,753(80.8)$ & $347(80.0)$ & \\
\hline Rural & 5,609 (24.4) & $87(20.0)$ & & 417 (19.2) & 87 (20.0) & \\
\hline Education level & & & $<0.01$ & & & 0.74 \\
\hline Elementary school or lower & $5,948(25.9)$ & $65(15.0)$ & & $323(14.9)$ & $65(15.0)$ & \\
\hline Middle school & $2,400(10.4)$ & $27(6.2)$ & & $147(6.8)$ & $27(6.2)$ & \\
\hline High school & $8,027(34.9)$ & $175(40.3)$ & & $922(42.5)$ & $175(40.3)$ & \\
\hline College & 6,633 (28.8) & 167 (38.5) & & $788(35.8)$ & 167 (38.5) & \\
\hline Monthly household income & & & 0.01 & & & 0.08 \\
\hline Low & 4,415 (19.2) & $63(14.5)$ & & $304(14.0)$ & $63(14.5)$ & \\
\hline Low medium & $5,818(25.3)$ & $110(25.4)$ & & $561(25.9)$ & $110(25.4)$ & \\
\hline Medium high & $6,400(27.8)$ & $115(26.5)$ & & $668(31.7)$ & $115(26.5)$ & \\
\hline High & $6,375(27.7)$ & $146(33.6)$ & & $617(23.4)$ & $146(33.6)$ & \\
\hline Marital status & & & $<0.01$ & & & 0.03 \\
\hline Married & $19,812(86.1)$ & $276(63.6)$ & & $1,499(69.1)$ & $276(63.6)$ & \\
\hline Unmarried & 246 (13.9) & $158(36.4)$ & & $671(30.9)$ & $158(36.4)$ & \\
\hline Occupation & & & $<0.01$ & & & 0.06 \\
\hline Professional & 2,808 (12.2) & 79 (18.2) & & $342(15.8)$ & 79 (18.2) & \\
\hline Managerial & $1,818(7.9)$ & 37 (8.5) & & $214(9.9)$ & 37 (8.5) & \\
\hline Semiskilled nonmanual & $2,843(12.4)$ & $76(17.5)$ & & $289(13.3)$ & 76 (17.5) & \\
\hline Semiskilled manual & 2,212 (9.6) & $25(5.8)$ & & $137(6.3)$ & $25(5.8)$ & \\
\hline Partly skilled & $2,175(9.4)$ & $31(7.1)$ & & $205(9.4)$ & $31(7.1)$ & \\
\hline Unskilled & $2,042(8.9)$ & $41(9.5)$ & & $165(7.6)$ & $41(9.5)$ & \\
\hline None & $9,110(39.6)$ & 145 (33.4) & & $818(37.7)$ & $145(33.4)$ & \\
\hline \multicolumn{7}{|l|}{ Clinical characteristics } \\
\hline Body mass index (kg/m²) & $23.61 \pm 3.37$ & $23.55 \pm 3.67$ & 0.73 & $23.22 \pm 3.55$ & $23.55 \pm 3.67$ & 0.08 \\
\hline Waist circumference (cm) & $81.00 \pm 9.97$ & $80.33 \pm 10.64$ & 0.17 & $79.32 \pm 10.33$ & $80.33 \pm 10.64$ & 0.06 \\
\hline Total caloric intake (kcal) & $1,918.65 \pm 834.02$ & $1,975.15 \pm 820.76$ & 0.16 & $1,980.44 \pm 853.0$ & $1,975.15 \pm 820.76$ & 0.91 \\
\hline Hypertension & $4,401(19.1)$ & $64(14.8)$ & 0.02 & $223(10.3)$ & $64(14.8)$ & $<0.01$ \\
\hline Diabetes mellitus & $1,577(6.9)$ & $20(4.6)$ & 0.06 & $84(3.9)$ & $20(4.6)$ & 0.47 \\
\hline Depressive symptoms & $2,849(12.4)$ & $70(16.1)$ & 0.02 & $225(11.8)$ & $70(16.1)$ & 0.01 \\
\hline
\end{tabular}

Values are presented as mean \pm standard error or number (\%). 
ses, 2,170 age- and sex-matched control subjects were included in a repeat analysis, the results of which are presented in the right column of Table 1.

Alcohol use, exercise status, and smoking status differed significantly between $\mathrm{AD}$ patients and unmatched control subjects, but these differences did not persist in the analysis involving matched control subjects. The proportions of AD patients who were nondrinkers $(20.2 \%, 88$ subjects) and nonsmokers (58.8\%, 255 subjects) were slightly lower relative to those of matched control subjects $(20.7 \%, 448$ subjects and $62.4 \%, 1,354$ subjects, respectively). In contrast, the proportion of $\mathrm{AD}$ patients who had engaged in exercise was slightly higher relative to that of matched control subjects ( $8.2 \%, 178$ subjects).

Of the variables representing socioeconomic status (residential area, educational level, monthly household income, marital status, and occupation), only marital status differed significantly between $\mathrm{AD}$ patients and matched control subjects; consequently, there was no significant difference in overall socioeconomic status between these groups. The proportion of married $\mathrm{AD}$ patients $(63.6 \%, 276$ subjects) was significantly lower relative to that of matched control subjects $(69.1 \%, 1,499$ subjects).

With respect to depressive symptoms of at least 2 weeks in duration and clinical characteristics (BMI $\left[\mathrm{kg} / \mathrm{m}^{2}\right]$, waist circumference $[\mathrm{cm}]$, total caloric intake [kcal], history of hypertension, and history of DM), depressive symptoms and history of hypertension differed significantly between $\mathrm{AD}$ patients and matched control subjects. The proportions of $\mathrm{AD}$ patients with depressive symptoms (16.1\%, 70 subjects) and a history of hypertension $(14.8 \%, 64$ subjects) were significantly higher relative to those of matched control subjects $(11.8 \%, 225$ subjects and $10.3 \%, 223$ subjects, respectively).

Table 2 shows the results of the multiple logistic regression analysis that included variables for which results of the descriptive analyses were significant, as independent variables, and presence of depressive symptoms of at least 2 weeks in duration as the dependent variable. Multiple logistic regression analyses were performed as follows: (1) unadjusted; (2) with alcohol

Table 2. Results of multiple logistic regression analysis showing the presence of depressive symptoms of at least 2 weeks in duration according to atopic dermatitis prevalence (unweighted data)

\begin{tabular}{rcc}
\hline & Control subjects & Atopic dermatitis \\
\hline Depression & & \\
Model $1^{*}$ & 1.00 & $1.46(1.09-1.95)$ \\
${\text { Model } 2^{\dagger}}_{\text {Model } 3^{\ddagger}}$ & 1.00 & $1.40(1.04-1.88)$ \\
\hline
\end{tabular}

Values are presented as odds ratios with 95\% confidence intervals in parentheses.

*Unadjusted, prevalence of atopic dermatitis in age- and sex-matched groups.

${ }^{\dagger}$ Alcohol use, exercise status, smoking status, and body mass index adjusted for.

${ }^{\ddagger}$ Alcohol use, exercise status, smoking status, marital status, occupation, body mass index, total caloric intake, and history of hypertension and diabetes mellitus adjusted for. use, exercise status, smoking status, and BMI adjusted for; and (3) with alcohol use, exercise status, smoking status, marital status, occupation, BMI, total caloric intake, history of hypertension, and history of DM adjusted for. In model 1, in which no adjustments were applied, the prevalence of depressive symptoms was significantly higher in $\mathrm{AD}$ patients (odds ratio, 1.46; 95\% confidence interval, 1.09-1.95), relative to that of matched control subjects. This difference persisted in model 2 (odds ratio, 1.40; 95\% confidence interval, 1.04-1.88). In model 3, all of the independent variables (alcohol use, exercise status, smoking status, marital status, occupation, BMI, total caloric intake, history of hypertension, and history of DM) were adjusted for, and the results of multiple logistic regression analysis showed an odds ratio of 1.36 (95\% confidence interval, 1.01-1.85), indicating that the proportion of $\mathrm{AD}$ patients with depressive symptoms was 1.36 times higher relative to that of matched control subjects.

\section{DISCUSSION}

In total, 23,442 subjects aged 19 years or older without a history of major medical illnesses (i.e., cancer, stroke, cardiovascular disease, tuberculosis, chronic obstructive pulmonary disease, bronchiectasis, chronic kidney disease, thyroid disease, and liver disease) or depressive disorders were selected from the KNHANES, and multiple logistic regression analyses were performed to establish the presence of depressive symptoms of at least 2 weeks in duration. Three models, adjusted for statistically significant differences in demographic, socioeconomic, and clinical characteristics (marital status and history of hypertension) were produced for $434 \mathrm{AD}$ patients and 2,170 age- and sex-matched control subjects. In all three models, levels of depressive symptoms in $\mathrm{AD}$ patients were significantly higher (odds ratios, 1.46, 1.40, and 1.36; 95\% confidence intervals, 1.09$1.95,1.04-1.88$, and 1.01-1.85, respectively) relative to those of matched control subjects.

Results of extensive research have shown that chronic skin diseases, such as $\mathrm{AD}$, which characteristically involve intense pruritus, cause psychosocial burden. Mental health disorders, including $\mathrm{ADHD}$ (attention deficit/hyperactivity disorder), depression, and autism, are known to be associated with $\mathrm{AD} .^{4)} \mathrm{In}$ addition, psychological disturbances, such as depression, anxiety, and suicidal ideation, and high levels of stigmatization and social withdrawal are associated with the disease. ${ }^{3,5,6)}$ The incidence of three mental health comorbidities, depression, anxiety, and suicidal ideation, had increased significantly in a 2015 study involving 4,994 individuals from 13 European countries. ${ }^{5}$ From this perspective, psychodermatology has recently published a number of studies that were conducted to improve both dermatological conditions and patients' quality of life. The monitoring of stress levels, depressive symptoms, suicidal ideation, 
and quality of sleep, which are important indices of mental health comorbidities and quality of life, should be taken into consideration and incorporated into management.

$\mathrm{AD}$ and clinical depression interact closely, and causal relationships between the two conditions have frequently been observed; for example, the onset or exacerbation of $\mathrm{AD}$ often follows stressful life events such as severe disease in a family member, divorce, or parental separation. ${ }^{7}$ In addition, $\mathrm{AD}$ patients, particularly those suffering from pruritus, experience psychological disturbances such as anxiety and depression. The following mechanisms could underlie this relationship: modulation of pruritus perception, ${ }^{8)}$ perturbation of epidermal permeability barrier homeostasis, ${ }^{9)}$ and acceleration of immune responses. ${ }^{10)}$ The acceleration of interactions between the two conditions occurs within a vicious cycle; therefore, all physicians, including both psychiatrists and nonpsychiatrists, should always consider mental health interventions (psychological, behavioral, and psychoeducational therapies and medications including antidepressants and anxiolytics) cautiously. Traditionally, other than conventional lifestyle modifications, little assistance is provided for $\mathrm{AD}$ patients in most clinics in Korea, but those interventions are considered important in controlling the dermatological condition in longstanding cases. It is particularly important that primary care physicians provide comprehensive, continuous long-term care.

Few studies have been conducted to examine mental health and quality of life in chronic dermatology patients in Korea; therefore, there is a definite need for further research to investigate the association between $\mathrm{AD}$ and mental health in sufficient numbers of adult patients and control subjects, using national data from the KNHANES. We selected depressive symptoms of at least 2 weeks in duration to represent general mental health, as this is one of diagnostic criteria for major depressive disorders in the fifth edition of the Diagnostic and Statistical Manual of Mental Disorders and one of the most common symptoms of stressful and depressive mental conditions. The proportion of $\mathrm{AD}$ patients with depressive symptoms was 1.36 times higher relative to that of control subjects. Many previous studies have involved pediatric patients; therefore, questions concerning mental health and quality of life were often answered by parents or other caregivers rather than the patients. In contrast, our study included a sufficient number of adult patients, who had suffered from chronic relapsing conditions and were able to assess their own mental health and quality of life accurately.

The only mental health variable used in the study was depressive symptoms of at least 2 weeks in duration, which did not appear to be strong enough for the diagnosis or indication of major depressive or anxiety disorders. It is also limited as the sole indicator for need for psychiatric or psychosocial interventions. Therefore, we future studies should include refined assessment tools, such as questions reflecting diagnostic criteria, the fifth edition of the Diagnostic and Statistical Manual of Mental Disorders, or severity assessments such as those of the SCORing Atopic Dermatitis Index., ${ }^{2,11)}$ In addition, attempts have recently been made to develop new psychosomatic scales (e.g., the Psychosomatic Diagnostic Criteria for Atopic Dermatitis and Psychosomatic Scale for Atopic Dermatitis, ${ }^{3,12,13)}$ which should be used in future studies. Studies examining the efficacy of various mental health interventions, such as those mentioned above, should be conducted in clinical settings and include Korean adults.

\section{CONFLICT OF INTEREST}

No potential conflict of interest relevant to this article was reported.

\section{REFERENCES}

1. Oh SH, Bae BG, Park CO, Noh JY, Park IH, Wu WH, et al. Association of stress with symptoms of atopic dermatitis. Acta Derm Venereol 2010; 90:582-8.

2. Ahn SG, Chang KH, Song JW, Chun SH. Common skin disease of Koreans (Korean). 2nd ed. Seoul: Doctor's Book; 2009.

3. Senra MS, Wollenberg A. Psychodermatological aspects of atopic dermatitis. Br J Dermatol 2014;170 Suppl 1:38-43.

4. Yaghmaie P, Koudelka CW, Simpson EL. Mental health comorbidity in patients with atopic dermatitis. J Allergy Clin Immunol 2013;131:428-33.

5. Dalgard FJ, Gieler U, Tomas-Aragones L, Lien L, Poot F, Jemec GB, et al. The psychological burden of skin diseases: a cross-sectional multicenter study among dermatological out-patients in 13 European countries. J Invest Dermatol 2015;135:984-91.

6. Simpson EL. Comorbidity in atopic dermatitis. Curr Dermatol Rep 2012;1:29-38.

7. Bockelbrink A, Heinrich J, Schafer I, Zutavern A, Borte M, Herbarth O, et al. Atopic eczema in children: another harmful sequel of divorce. Allergy 2006;61:1397-402.

8. Gupta MA, Gupta AK. Depression modulates pruritus perception: a study of pruritus in psoriasis, atopic dermatitis and chronic idiopathic urticaria. Ann N Y Acad Sci 1999;885:394-5.

9. Garg A, Chren MM, Sands LP, Matsui MS, Marenus KD, Feingold KR, et al. Psychological stress perturbs epidermal permeability barrier homeostasis: implications for the pathogenesis of stress-associated skin disorders. Arch Dermatol 2001;137:53-9.

10. Hashizume H, Horibe T, Ohshima A, Ito T, Yagi H, Takigawa M. Anxiety accelerates T-helper 2-tilted immune responses in patients with atopic dermatitis. Br J Dermatol 2005;152:1161-4.

11. Slattery MJ, Essex MJ, Paletz EM, Vanness ER, Infante M, Rogers GM, et al. Depression, anxiety, and dermatologic quality of life in adolescents with atopic dermatitis. J Allergy Clin Immunol 2011;128:668-71.

12. Ando T, Hashiro M, Noda K, Adachi J, Hosoya R, Kamide R, et al. Development and validation of the psychosomatic scale for atopic dermatitis in adults. J Dermatol 2006;33:439-50.

13. Kelsay K, Klinnert M, Bender B. Addressing psychosocial aspects of atopic dermatitis. Immunol Allergy Clin North Am 2010;30:385-96. 